\title{
Appendix of Generalizing DET curves across application scenarios
}

\author{
Norman Poh and Chi Ho Chan
}

\section{Summary of 4 Data SETS AND 13 USE-CASES}

TABLE I: The data set and scenarios used to benchmark the quality of prediction in terms of coverage

(a) Four score data sets used to measure coverage

\begin{tabular}{|c|c|c|c|c|c|c|c|}
\hline Scenario & Case & $\begin{array}{l}\text { Prior ratio, } \\
\operatorname{Prob}\left(Q \mid \omega_{1}\right)\end{array}$ & $\begin{array}{l}\text { Prior } \\
\text { ratio, } \\
\operatorname{Prob}\left(Q \mid \omega_{0}\right)\end{array}$ & \multirow{2}{*}{\multicolumn{4}{|c|}{ (b) 13 cases for assessing coverage }} \\
\hline \multirow{3}{*}{$\begin{array}{l}\text { Zero and spoof } \\
\text { attack }\end{array}$} & $\omega_{1}$ & Genuine comparison, & Similarity & & & & \\
\hline & $\omega_{0}$ & $\begin{array}{l}\text { N/A, N/A, N/A, N/A, N/A } \\
\text { Zero-effort impostor } \\
\text { comparison, EcoFlex, }\end{array}$ & $\begin{array}{l}\text { score with } \\
\text { Biometrika } \\
\text { sensor, }\end{array}$ & Scenario & Case & $\begin{array}{l}\text { Prior } \\
\text { ratio, } \\
\text { Prob }\left(Q \mid \omega_{1}\right)\end{array}$ & $\begin{array}{l}\text { Prior } \\
\text { ratio, } \\
\text { Prob }\left(Q \mid \omega_{0}\right)\end{array}$ \\
\hline & & $\begin{array}{l}\text { Gelatine, Latex, } \\
\text { Silgum, WoodGlue }\end{array}$ & & \multirow{3}{*}{$\begin{array}{l}\text { Zero and spoof } \\
\text { attack } \\
\text { (Biometrika) }\end{array}$} & $\begin{array}{l}\text { Zero-effort } \\
\text { attack }\end{array}$ & $\begin{array}{llll}{\left[\begin{array}{llll}1 & 0 & 0 & 0 \\
0 & 0\end{array}\right]} & & \end{array}$ & 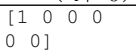 \\
\hline \multirow[t]{3}{*}{$\begin{array}{l}\text { Zero and spoof } \\
\text { attack }\end{array}$} & $\omega_{1}$ & $\begin{array}{l}\text { Genuine comparison, } \\
\text { N/A, N/A, N/A, N/A, N/A }\end{array}$ & \multirow{3}{*}{$\begin{array}{l}\text { Similarity } \\
\text { score with } \\
\text { Italdata } \\
\text { sensor, } \\
|Q|=6\end{array}$} & & Spoof attack & $\begin{array}{llll}{\left[\begin{array}{llll}1 & 0 & 0 & 0 \\
0 & 0\end{array}\right]} & & \end{array}$ & $\begin{array}{llll}{\left[\begin{array}{llll}0 & 1 & 1 & 1 \\
1 & 1\end{array}\right]} & & \\
\end{array}$ \\
\hline & \multirow[t]{2}{*}{$\omega_{0}$} & \multirow{2}{*}{$\begin{array}{l}\text { Zero-effort impostor } \\
\text { comparison, EcoFlex, } \\
\text { Gelatine, Latex, } \\
\text { Silgum, WoodGlue }\end{array}$} & & & $\begin{array}{l}\text { Balanced } \\
\text { attack }\end{array}$ & $\begin{array}{llll}{\left[\begin{array}{llll}1 & 0 & 0 & 0 \\
0 & 0\end{array}\right]} & & \end{array}$ & $\begin{array}{lrrr}{\left[\begin{array}{llll}5 & 1 & 1 & 1 \\
1 & 1\end{array}\right]} & & \\
\end{array}$ \\
\hline & & & & \multirow{3}{*}{$\begin{array}{l}\text { Zero and spoof } \\
\text { attack } \\
\text { (Italdata) }\end{array}$} & $\begin{array}{l}\text { Zero-effort } \\
\text { attack }\end{array}$ & $\begin{array}{llll}{\left[\begin{array}{llll}1 & 0 & 0 & 0 \\
0 & 0\end{array}\right]} & & \end{array}$ & 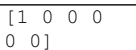 \\
\hline \multirow[t]{6}{*}{ Cross-sensor } & \multirow[t]{3}{*}{$\omega_{1}$} & \multirow{3}{*}{$\begin{array}{l}\text { Genuine comparison with } \\
\text { Biometrika sensor, } \\
\text { Genuine comparison } \\
\text { with Italdata sensor, } \\
\text { Cross-sensor genuine } \\
\text { comparison }\end{array}$} & \multirow{6}{*}{$\begin{array}{l}\text { Cross-sensor } \\
\text { comparison } \\
\text { involving } \\
\text { two sensors, } \\
|Q|=3\end{array}$} & & Spoof attack & $\begin{array}{llll}{\left[\begin{array}{llll}1 & 0 & 0 & 0 \\
0 & 0\end{array}\right]} & & \end{array}$ & 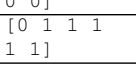 \\
\hline & & & & & $\begin{array}{l}\text { Balanced } \\
\text { attack }\end{array}$ & $\begin{array}{llll}{\left[\begin{array}{llll}1 & 0 & 0 & 0 \\
0 & 0\end{array}\right]} & & \end{array}$ & 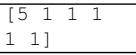 \\
\hline & & & & \multirow{4}{*}{ Cross-sensor } & $\begin{array}{l}\text { Biometrika } \\
\text { sensor }\end{array}$ & {$\left[\begin{array}{lll}1 & 0 & 0\end{array}\right]$} & {$\left[\begin{array}{lll}1 & 0 & 0\end{array}\right]$} \\
\hline & \multirow[t]{3}{*}{$\omega_{0}$} & \multirow{3}{*}{$\begin{array}{l}\text { Zero-effort non-match } \\
\text { comparison involving } \\
\text { Biometricka sensor, } \\
\text { Italdata sensor, and } \\
\text { cross-sensor }\end{array}$} & & & $\begin{array}{l}\text { Italdata } \\
\text { sensor }\end{array}$ & {$\left[\begin{array}{lll}0 & 1 & 0\end{array}\right]$} & {$\left[\begin{array}{lll}0 & 1 & 0\end{array}\right]$} \\
\hline & & & & & Both sensor & {$\left[\begin{array}{lll}1 & 1 & 0\end{array}\right]$} & {$\left[\begin{array}{lll}1 & 1 & 0\end{array}\right]$} \\
\hline & & & & & Cross sensor & {$\left[\begin{array}{lll}1 & 1 & 1\end{array}\right]$} & {$\left[\begin{array}{lll}1 & 1 & 1\end{array}\right]$} \\
\hline \multirow{5}{*}{$\begin{array}{l}\text { Varying } \\
\text { quality }\end{array}$} & \multirow[t]{3}{*}{$\omega_{1}$} & \multirow{3}{*}{$\begin{array}{l}\text { Genuine comparison } \\
\text { conditional on NFIQ } \\
\text { quality levels } 1-3 \text { and } \\
\text { combined levels of } 4 \\
\text { and } 5\end{array}$} & \multirow{5}{*}{$\begin{array}{l}\text { Match and } \\
\text { non-match } \\
\text { comparisons } \\
\text { conditional } \\
\text { upon } \\
\text { different } \\
\text { sample } \\
\text { quality } \\
\text { levels, } \\
|Q|=4\end{array}$} & \multirow[t]{4}{*}{ Varying quality } & $\begin{array}{l}\text { High quality } \\
\text { tendency }\end{array}$ & {$\left[\begin{array}{llll}8 & 4 & 2 & 1\end{array}\right]$} & {$\left[\begin{array}{llll}8 & 4 & 2 & 1\end{array}\right]$} \\
\hline & & & & & $\begin{array}{l}\text { Low quality } \\
\text { tendency }\end{array}$ & {$\left[\begin{array}{llll}1 & 2 & 4 & 8\end{array}\right]$} & {$\left[\begin{array}{llll}1 & 2 & 4 & 8\end{array}\right]$} \\
\hline & & & & & $\begin{array}{l}\text { Equal } \\
\text { guality }\end{array}$ & {$\left[\begin{array}{llll}1 & 1 & 1 & 1\end{array}\right]$} & {$\left[\begin{array}{llll}1 & 1 & 1 & 1\end{array}\right]$} \\
\hline & $\omega_{0}$ & $\begin{array}{l}\text { Non-match zero-effort } \\
\text { attack comparison }\end{array}$ & & & $\begin{array}{l}\text { quality } \\
\text { tendency }\end{array}$ & & \\
\hline & & $\begin{array}{l}\text { conditional on NFIQ } \\
\text { quality levels } 1-3 \text { and } \\
\text { combined levels of } 4 \\
\text { and } 5\end{array}$ & & & & & \\
\hline
\end{tabular}

\section{CASE DEMOnSTRATions}

In this section, a demonstration of the derived DET confidence intervals of another two applications is given, namely: assessing biometric performance with zero-effort and spoof attack; and assessing biometric performance with two independent and cross sensors.

(i) Spoof and zero-effort attacks: Figure 1(a) shows the predicted DET curves with confidence intervals of three use-case scenarios, namely, balanced attack, spoof attack, and zero-effort attack. As can be observed, the zero-effort attack has the lowest error rates because the curve (with confidence intervals) is close to the origin of (FMR, FNMR). The spoof attack, on the other hand, has the highest error rate. In comparison, the balanced attack has a DET curve, with confidence intervals, lie somewhere between these two curves.

We also plotted the estimated $c d f$ for the different score sets in Figure 1(b). The $c d f$ of the genuine match comparison is an increasing function of the decision threshold (the $\mathrm{X}$-axis) which resides in the score space. The zero-effort non-match comparison has the steepest curve, thus, explaining the good performance in shown in Figure 1(b). The spoof samples are significantly larger in value, as shown by their $1-c d f \mathrm{~s}$ as a decreasing function 
of the decision threshold. This function, that is, FMR, is decreasing because as one increases the decision threshold, more spoof samples are rejected. However, this comes at the expense of increased false rejections, as measured by FNMR. The increase in error is reflected by the spoof DET curve shown in Figure 1.

(a) FMRs and FNMRs

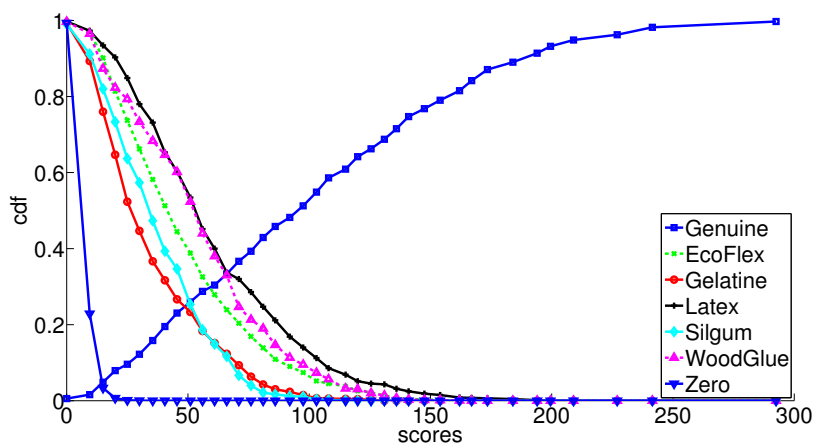

(b) Balanced attack

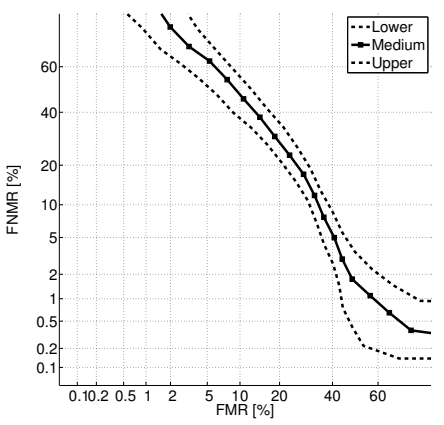

(c) Spoof attack

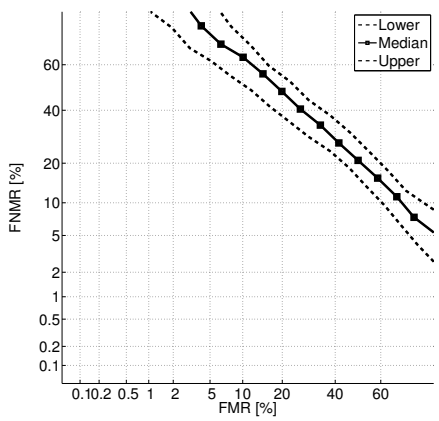

(d) Zero-effort impersonation

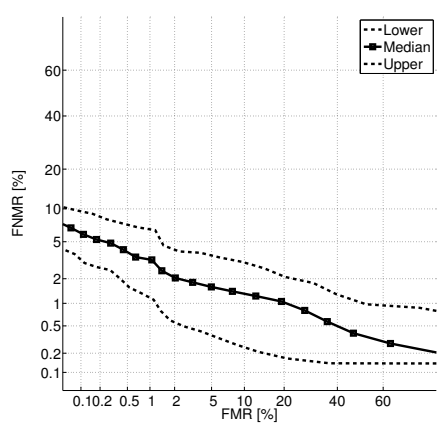

Fig. 1: (a) The false match rate curves $(1-c d f)$ due to spoof attacks induced by five different spoof fabrication materials, as well as the zero-effort attack. These curves constitute the negative class and are decreasing functions of the similarity score. The false non-match rate $(c d f)$ of the genuine (match) comparison is shown as an increasing function of the similarity score. (b)-(d): Three sample of curves. The curve "zero" is plotted with $\left\{y \mid \omega_{0}, Q=\right.$ Zero $\}$ as a negative class against $\left\{y \mid \omega_{0}, Q=\right.$ Genuine $\}$ as the positive class. The curve "spoof" is plotted with $\left\{y \mid \omega_{0}, Q=\right.$ Spoof $\}$ where the spoof materials could be any of the five materials with weight $w_{\text {neg }}$ whose ratio is set to be equal, i.e., $[1,1,1,1,1]$. The "balanced" curve is plotted with the five spoofing materials and the zero-effort attack whose prior weight vector is set to the ratio of $[5,1,1,1,1,1]$ so that the prior probability of a zero effort attack versus any of the spoof attack is equal.

(ii) Two independent and cross sensors: we will have three sets of match comparison. In this data set, we have two sensors, namely Biometrika and Italdata sensors. We, therefore, have match comparisons due to the comparison of template and query samples of one sensor, another sensor, as well as their cross comparison wherein the template has been captured using one sensor; and the query, with another sensor. As for the non-match comparisons, we observed that the $c d f$ does not change with the sensor type. For this reason, we consider only the non-match comparison due to each of the two sensors. The five $c d f$ s are shown in Figure 2(a).

We then identify four scenarios, and in each scenario, we weigh the priors of the five $c d f$ s differently. The priors are listed in Table I(b). The DET curves for these four scenarios are shown in Figures 2(b)-(e), respectively. As can be observed, the last scenario which involves cross-device comparison has a significantly worst performance than the first three scenarios which do not involve two types of single-device comparison. 
(a) FMRs and FNMRs

(b) Biometrika only sensor

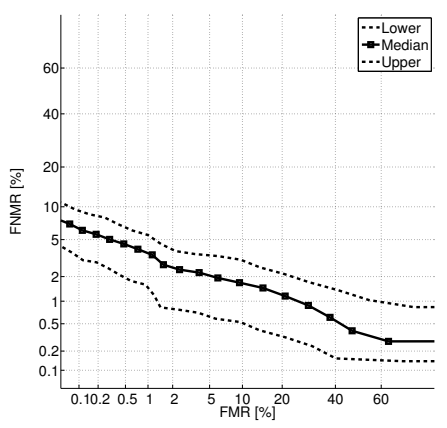

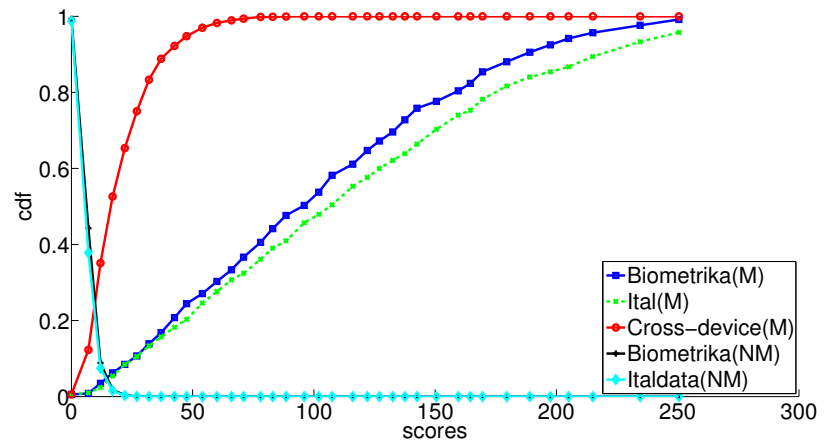

(c) Italdata only sensor

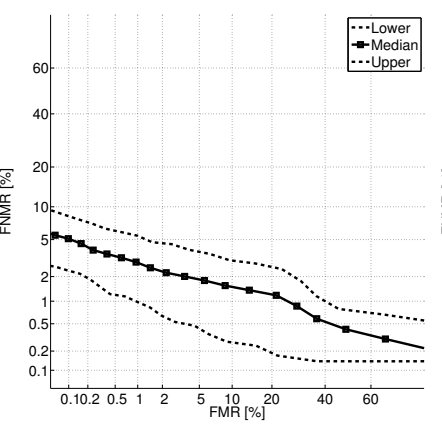

(d) Both sensors

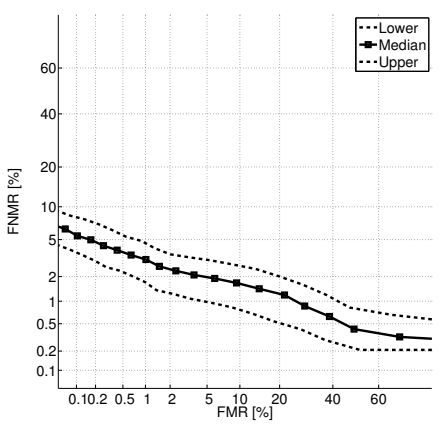

(e) Cross-device comparison

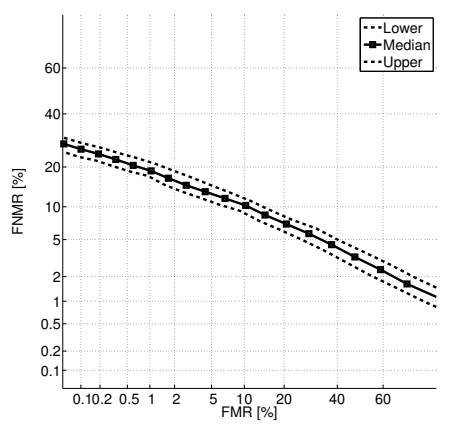

Fig. 2: The FMRS and FNMRS for the cross-sensor setting. In the legend, M indicates match comparisons (postive class) whereas NM indicates NM comparisons (negative class). The DET curves of four use-cases: (b) Biometrika only sensor, (c) Italdata only sensor, (d) both sensors, (e) cross-device comparison. 